\section{Regards sur l'économie allemande}

Bulletin économique du CIRAC

112 | 2014

Varia

\title{
Les deux visions de l'Europe qu'ont les Allemands
}

\section{Thomas Petersen}

\section{OpenEdition}

\section{Journals}

Édition électronique

URL : http://journals.openedition.org/rea/4658

DOI : 10.4000/rea.4658

ISBN : 978-2-8218-0826-3

ISSN : 1965-0787

Éditeur

CIRAC

Édition imprimée

Date de publication : 28 avril 2014

Pagination : 25-34

ISSN : 1156-8992

Référence électronique

Thomas Petersen, "Les deux visions de l'Europe qu'ont les Allemands », Regards sur l'économie allemande [En ligne], 112 | avril 2014, mis en ligne le 01 avril 2016, consulté le 01 mai 2019. URL http://journals.openedition.org/rea/4658 ; DOI : 10.4000/rea.4658 


\section{Les deux visions de l'Europe qu'ont les Allemands}

\section{Thomas Petersen}

\begin{abstract}
Aristide Briand et Gustav Stresemann, Konrad Adenauer et Robert Schuman pouvaient-ils seulement imaginer qu'un jour, certains considéreraient l'intégration européenne comme une menace pour la liberté ? C'était peu probable à l'époque. Mais pas aujourd'hui, si on en croit le débat public en Allemagne. Le symptôme le plus révélateur en est la fulgurante montée en puissance du parti Alternative für Deutschland (AfD). Quelques mois seulement après sa création il $y$ a tout juste un an, il a remporté $4,7 \%$ des voix lors des élections au Bundestag, le 22 septembre 2013, ne ratant que de peu la barre des $5 \%$ nécessaires pour entrer au Bundestag. La probabilité est grande de voir l'AfD représenté au Parlement européen à l'issue des élections du 25 mai de cette année.
\end{abstract}

Comme son rejet de l'intégration européenne dans sa configuration actuelle, et surtout de l'Euro, est souvent défendu par des arguments à l'emporte-pièce, ce parti se voit reprocher par nombre de ses contradicteurs politiques d'être un parti radical de droite, en quelque sorte l'équivalent allemand du Front national français. Mais c'est passer à côté de l'essentiel. Certes, nombre de thèses défendues par l'AfD relèvent du populisme de droite, mais son programme se fonde surtout sur la doctrine libérale. Son président, l'économiste Bernd Lucke, est professeur à l'Université de Hambourg, et l'un de ses plus éminents candidats au Parlement européen est Hans-Olaf Henkel, ancien président de la Fédération allemande de l'industrie (BDI). Aucun des deux ne peut être soupçonné d'extrémisme. Et au parti AfD, rien de comparable non plus au nationalisme agressif d'un FN.

Ses thèses et son électorat sont ancrés au centre du spectre politique. Autrement dit, les doutes profonds sur l'intégration européenne exprimés par l'AfD sont ceux qui animent aujourd'hui le centre pourtant traditionnellement pro-européen de la société allemande. Au cœur de son euroscepticisme se trouve la hantise que l'Union européenne soit une construction centralisée, souffrant d'un manque de légitimation démocratique et bridant tant la souveraineté de ses Etats membres que la liberté d'action individuelle de leurs citoyens.

La question qui se pose dès lors est de savoir si une telle approche est largement partagée par les Allemands ou si, à terme, elle ne reflètera que l'opinion d'une petite minorité. Pour répondre à cette question, il faut élargir le champ.

\section{L'idée d'Europe et la liberté}

Lorsqu'on jette un rapide coup d'œil sur l'histoire de l'évolution du processus menant à la naissance de l'Union européenne, il semble à première vue évident de supposer que l'idée de liberté était la force motrice de l'idée d'Europe. Après tout, ce sont des Etats libres de l'Europe occidentale qui se sont réunis après la Seconde Guerre mondiale au sein d'une communauté d'abord économique puis politique. Ce processus d'intégration était généralement mû par l'objectif de la libre circulation des biens.

Mais si on regarde de plus près les arguments avancés au $X X^{e}$ siècle pour promouvoir l'intégration européenne, alors on s'aperçoit que la liberté - comprise comme la valeur au fondement du modèle de société - n'y joue qu'un rôle marginal. Elle est certes posée, tantôt explicitement, tantôt implicitement, comme le

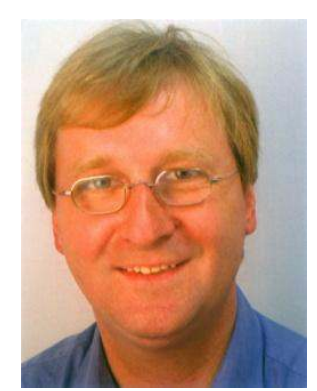

Thomas Petersen, docteur en sciences de la communication, est chercheur à l'Institut für Demoskopie d'Allensbach. Il enseigne également à l'Université de Mayence et préside la World Association for Public Opinion Research (WAPOR). Dans le quotidien F.A.Z., il publie régulièrement les "Allensbach-Analysen " sur l'état de l'opinion.

A l'origine de l'idée d'Europe : la libre circulation des biens ?

Plutôt la paix, grâce à un cadre contraignant 
Entente franco-allemande : préalable et condition sine qua non

Institutions communes d'abord union des peuples ensuite

La CECA pour rendre impossible tout réarmement

Interdépendance financière dès avant l'Euro fondement même du processus d'intégration, s'assimilant de ce fait à une sorte de bruit de fond. Mais depuis les débuts de l'intégration européenne, tant ses précurseurs que ses artisans ont posé d'autres priorités : l'intégration semble avoir été pour eux avant tout un acte d'auto-affirmation du continent face aux autres puissances, ainsi qu'une œuvre de paix que seul rendaient possible un cadre régissant la vie en communauté, avec son contrôle mutuel, celui-ci se soldant par la réduction des libertés à l'échelon national.

La force destructrice des conflits intra-européens, surtout le traumatisme des deux guerres mondiales, conjugués au sentiment des Européens d'avoir perdu toute influence sur la scène politique mondiale, constituent après 1945 l'arrièreplan des efforts consacrés à une union en Europe et tout particulièrement à une entente franco-allemande. Ainsi, en mars 1950, Konrad Adenauer expliquait au journaliste américain Howard Kingsbury Smith : " une union entre la France et l'Allemagne permettrait à une Europe gravement malade de renouer avec la vie et de reprendre un nouvel élan. Au plan psychologique et matériel, elle pourrait déployer une énorme influence et libérer des forces qui sauront assurément sauver l'Europe. Je crois que c'est là la seule possibilité pour atteindre l'union de l'Europe. Car ainsi disparaîtrait la vision de la rivalité entre ces deux pays ». En mars 1950 également, Charles de Gaulle réagissait à la proposition d'une union franco-allemande avec le pathos qui lui était propre, mais il partageait cette approche sur le fond. Adenauer résume ainsi la réaction de de Gaulle : « il rappela qu'Attila fut battu aux Champs Catalauniques grâce à l'effort conjugué des Gaulois, des Germains et des Romains. Une union entre la France et l'Allemagne prolongerait l'œuvre de Charlemagne... et il se dit très impressionné par la puissance inhérente à une telle démarche. L'avenir de l'Europe dépend dans une très large mesure de l'évolution des relations franco-allemandes ".

Quand, aujourd'hui, certaines voix s'élèvent pour regretter que l'Europe unie ne soit finalement qu'une Europe des institutions (il est intéressant de relever qu'elles présupposent alors que ce fait entre en contradiction avec les intérêts des citoyens), il est bon de rappeler que c'était là l'objectif premier. Plus récemment, en 1996, François Mitterrand écrivait : "Je ne me défais pas ... de l'idée qu'une société ne survit que par ses institutions. Ainsi en sera-t-il de l'Europe. Puisque tout jusqu'ici repose sur la force qui ne cède elle-même qu'à la violence, cassons cette logique et substituons-lui celle du libre contrat. Si la communauté, fille de la raison, adopte des structures durables, vainqueur, vaincu, ces notions appartiendront à notre préhistoire. L'exiguïté de notre continent, ... le besoin éprouvé à l'est comme à l'ouest de l'Europe d'exister et de peser sur le destin de la planète en écartant l'étau qui, d'Asie et d'Amérique, se resserre sur nous, concourent à cette prise de conscience ». Pour Mitterrand, donc, la priorité était la création d'institutions communes; elle était le préalable pour que, ensuite, les peuples puissent s'unir au plan émotionnel.

Déjà, lors de la création de la Communauté du charbon et de l'acier (CECA), Robert Schuman et Konrad Adenauer étaient mus par l'idée que, pour rendre impossible toute nouvelle guerre, il faut littéralement enchaîner les uns aux autres les peuples européens via les institutions. Schumann pour sa part n'a jamais fait mystère du fait que sa vision d'une gestion commune du charbon et de l'acier ne reposait pas sur la confiance, mais sur la méfiance vis-à-vis de l'Allemagne. A cette époque, nombreuses étaient les craintes françaises de voir l'Allemagne, une fois ses forces recouvrées, attaquer une nouvelle fois la France. Le contrôle conjoint de la sidérurgie et des industries minières devait rendre impossible à l'Allemagne tout réarmement à l'abri des regards français.

Et c'est dans la fidélité à cette approche ancrée dans l'histoire que s'inscrivent les étapes suivantes de l'intégration, avec leur lot d'abandon d'éléments de souveraineté nationale à des institutions européennes et la prise d'engagements mutuels - y compris budgétaires - au sein de l'Union. Et dans cette évolution, l'adoption de la monnaie unique n'a rien d'une césure. A cet égard, ce que rapporte Mitterrand de sa première rencontre en qualité de président de la 
République avec le chancelier de la République fédérale Helmut Schmidt en 1981 est révélateur de l'état de l'interdépendance des politiques budgétaires comme du degré d'indépendance des banques centrales dès le début des années 1980: "Le Chancelier promit son soutien à la monnaie française et la Bundesbank acheta quelques centaines de millions de francs. II est vrai que l'économie allemande aurait eu du mal à s'accommoder d'une tempête monétaire qui eût mis le système européen en péril ».

Cet exemple montre bien combien, longtemps avant la création de l'Euro, les économies européennes étaient interdépendantes au point que s'était installée une sorte de communauté reposant sur la responsabilité mutuelle, autrement dit cette forme de dépendance économique si violemment rejetée aujourd'hui en Allemagne parce qu'on y craint d'avoir un jour à payer les dettes d'autres Etats membres et d'avoir à en porter finalement le préjudice.

II faut bien convenir aussi que cette thématique a considérablement gagné en actualité depuis la crise de la dette souveraine en Grèce et dans d'autres Etats de la zone Euro ou qu'elle s'est pour le moins hissée plus nettement dans la conscience des Allemands. Au vu de cette évolution, il est dès lors parfaitement plausible de supposer que l'image de l'Union européenne pourrait prendre une teinte globalement négative dans l'esprit des citoyens allemands - et ce, potentiellement bien au-delà de la naissance d'un minuscule parti d'eurosceptiques comme l'AfD. II se pourrait que les Allemands aient plus largement que par le passé pris conscience de l'ampleur des transferts de souveraineté qu'ils ont consentis à l'échelon communautaire. Conjugués à cela, les griefs récurrents formulés depuis de longues années dans l'espace public et incriminant un soidisant déficit de démocratie des institutions européennes ou l'ingérence en apparence croissante d'une administration communautaire bureaucratique dans le processus législatif des Etats membres pourraient aisément laisser croire que indépendamment de l'impact positif qu'il peut avoir dans d'autres domaines - le processus d'intégration européenne est perçu par de nombreux Allemands comme une grave mise en danger de la liberté de l'individu. Cette lecture retrace-t-elle la réalité des faits ? Pour répondre à cette question, il convient d'abord de se demander comment a évolué l'image de l'Europe des Allemands dans les décennies précédant la crise de la finance mondiale.

\section{Un processus rampant de distanciation vis-à-vis de l'Europe}

Les tout premiers sondages de I'Institut für Demoskopie d'Allensbach (IfD) avaient été menés auprès de jeunes Allemands de 15 à 25 ans. Ils avaient été commandités par le gouvernement militaire français de la zone sud-ouest de l'Allemagne qui s'inquiétait de savoir s'il serait possible de construire une société civile pacifique avec cette jeune génération qui avait grandi sous la dictature et durant la guerre. Quelle image du monde, quelles opinions politiques, quelle vision de la vie avaient ces jeunes ? Autant de questions qu'il n'est possible d'aborder que grâce aux moyens de la démoscopie (voir REA 80/2007). Le 8 mai 1947, l'IfD lança donc ses premiers entretiens-tests. C'est ainsi qu'est née en Allemagne l'étude de l'opinion publique.

A travers les réponses à ces sondages se dessinait un état déprimé, résigné, de l'opinion. "Pensez-vous qu'on puisse généralement faire confiance aux gens ? ". A cette question inspirée des questionnaires de l'Institut Gallup aux Etats-Unis, $69 \%$ des jeunes Allemands avaient répondu «non ", seulement $16 \%$ «oui ». A la question: "pensez-vous qu'au cours des cinq prochaines années une nouvelle guerre va éclater? », $62 \%$ avaient répondu « oui ». C'est donc en toute logique que $38 \%$ aient répondu " oui » à la question : "Souhaiteriez-vous quitter l'Allemagne maintenant ou plus tard ? ". Quant à la politique, elle intéressait peu les jeunes. A la question : "vous intéressez-vous aux partis politiques actuels ? », $72 \%$ avaient dit «non ». Ils n'attendaient rien du politique et lui avaient tourné le dos.
L'intégration européenne, menace pour la liberté des individus?

La vision du monde des jeunes Allemands en 1947

Résignation et défiance vis-à-vis du politique 
Mais dans cette sombre humeur, les réponses à une courte série de questions sur l'Europe laissaient passer comme un rayon de soleil. La première d'entre elles était: "On entend aujourd'hui dire certains que les Etats européens devraient s'unir pour former des Etats-Unis d'Europe. Avez-vous déjà entendu parler de cette idée ? " $60 \%$ des jeunes sondés affirmaient en avoir entendu parler. A la question suivante, à savoir : "Cette idée vous semble-t-elle intéressante ou non ? ", $29 \%$ déclaraient ne pas la trouver intéressante, une majorité relative de $40 \%$ se montrait intéressée, et même de $66 \%$ parmi les étudiants interrogés. En revanche, seuls $21 \%$ des jeunes s'intéressaient aux nouveaux partis politiques qui venaient de voir le jour. Enfin, on leur avait demandé : «Si la constitution d'Etats-Unis d'Europe devait être votée aujourd'hui, seriez-vous pour, contre, ou cela vous laisserait-il indifférent ? ».50\% se dirent « pour ».

Il est compréhensible que, dans une Allemagne vaincue, occupée et morcelée, tant de gens voient dans une construction supra-nationale telle que les «EtatsUnis d'Europe " la seule perspective d'avenir quelque peu réjouissante. Mais même après la constitution de la République fédérale d'Allemagne (2 mai 1949) et après les premiers pas effectifs sur la voie d'une intégration européenne, la population allemande a gardé longtemps cet enthousiasme prononcé à l'idée d'une Europe unie.

Depuis, Les réactions actuelles des sondés aux questions traitant de la thématique eu-

ropéenne n'ont plus rien de comparable avec celles des débuts, ni même avec celles des années 1960 et 1970. L'enthousiasme d'antan a totalement disparu ; dès avant qu'éclate la crise de la dette souveraine, il avait cédé la place non pas à un rejet, mais à un manque d'intérêt généralisé. Et contrairement à ce qu'on croit souvent, ce n'était pas la génération la plus ancienne, mais surtout celle des jeunes que le thème de l'Europe laissait indifférente.

Le graphique 1 montre le schéma des réponses caractéristique des années avant la crise à travers la question : "Vous intéressez-vous à la politique européenne - je veux dire aux décisions qui sont prises à Bruxelles ou au Parlement européen de Strasbourg - ou ne vous intéresse-t-elle pas ? ». En mai 2008, les trois quarts des moins de 30 ans disaient que ce qui se passe à Bruxelles ou à Strasbourg ne les intéresse " pas beaucoup » ou " pas du tout »; le taux n'était 'que' de $59 \%$ chez les 60 ans et plus. "Elle m'intéresse » réunissait $25 \%$ des réponses des jeunes et $41 \%$ de celles des plus âgés.

\section{Graphique 1. Avant la crise, déjà, la jeune génération montrait un faible intérêt pour la politique européenne.}

Question : "Vous intéressez-vous à la politique européenne - je veux dire aux décisions qui sont prises à Bruxelles ou au Parlement européen de Strasbourg - ou ne vous intéresse-t-elle pas ? " (Réponses en \%)

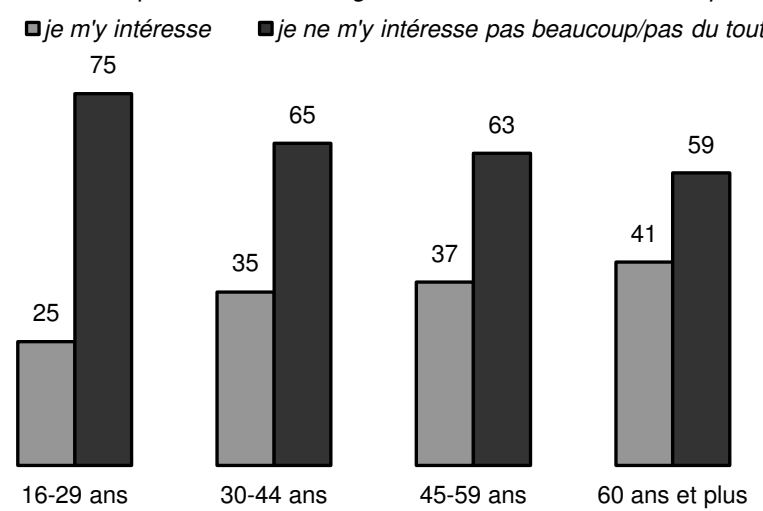

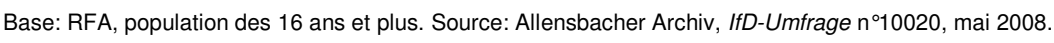

Il faut se rendre à l'évidence : les forces grâce auxquelles ont pu naître après la Seconde Guerre mondiale la CECA et le marché commun disparaissent vite. L'expérience traumatique qui animait les survivants à la Seconde Guerre mondiale et les poussait à tout mettre en œuvre pour que le continent ne puisse 
plus jamais céder aux sirènes du nationalisme n'est pas partagé par leurs petits-enfants ; c'est dans la nature des choses.

Quand, dans les années 1990, le chancelier Helmut Kohl rappelait avec insistance que l'intégration européenne est une question de guerre et de paix, et quand, dans son dernier grand discours devant les députés du Parlement européen, le président Mitterrand leur assénait cette phrase : «le nationalisme, c'est la guerre!", nombreux déjà étaient ceux qui, à l'époque, considéraient cela comme quelque peu démodé. Aujourd'hui, ces messages n'atteignent plus la jeune génération. Dans une interview parue en juin 2006 dans le quotidien Frankfurter Allgemeine Zeitung, Wolfgang Schüssel, alors chancelier de la République autrichienne, avait bien résumé le problème: "L'Europe, avait-il dit, doit à chaque fois être posée sur de nouveaux fondements. Les gens ont un besoin impérieux qu'on leur présente l'Europe autrement. Ils ne veulent pas qu'on les ennuie avec des discussions sur les méthodes ou des différends sur les procédures, ils veulent au contraire qu'on leur explique le message clé de l'Europe. Et celui-ci est et reste avant tout un message de paix. Allez dans le cimetière d'un village regarder les tombes des victimes des guerres mondiales, et vous comprendrez ce que je veux dire. Ce message-là ne va plus de soi, et les jeunes ne le connaissent plus. L'Europe ne doit pas se légitimer seulement par l'économie. L'Europe était naguère un thème qui touchait le cœur : 'plus jamais la guerre !'. Aujourd'hui, c'est un thème qui touche l'intellect, ou alors il s'est dégradé et touche le portemonnaie : que m'apporte-t-elle ? quel bénéfice puis-je en tirer? Cette approche est mortelle ».

Le refroidissement de l'enthousiasme européen des Allemands est un processus rampant. Au fur et à mesure que le rêve de l'unité de l'Europe devenait réalité, le processus d'intégration se faisait à la fois plus quotidien et plus complexe. Les questions de fond furent reléguées à l'arrière-plan par des questions touchant la vie de chacun : quotas de pêche, subventions agricoles ou contributions des Etats membres.

Plus récemment, cette évolution a été forcée par le fait que presque toutes les grandes étapes de l'intégration au cours de ces dernières années - le Traité de Maastricht et l'adoption de l'Euro, l'élargissement de l'UE à l'est et l'ouverture des négociations d'adhésion de la Turquie - se sont effectuées contre la volonté de la majorité, du moins de celle des Allemands. Sans nul doute possible, les décisions concernant l'élargissement à l'est et l'ouverture de négociations avec la Turquie ont présumé des forces des Allemands.

Graphique 2. Avec quelle rapidité devrait être atteinte l'union de l'Europe ?

Question : "A quelle vitesse devrait se faire l'évolution vers une Europe unie ? Plus vite, moins vite, ou à la même vitesse que jusqu'ici ? » (Réponses en \%)

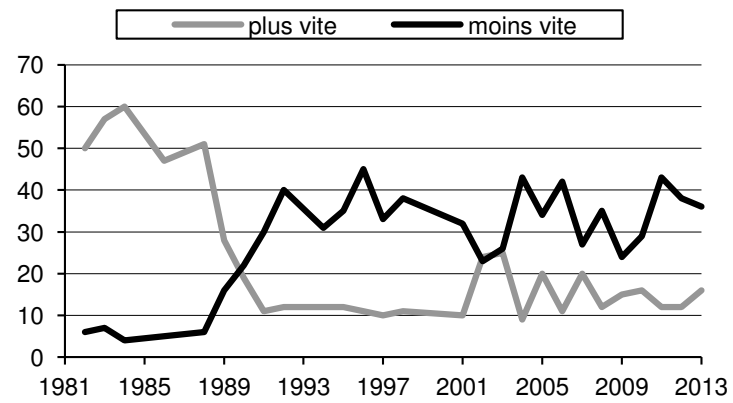

Source : Allensbacher Archiv, IfD-Umfragen, dernier sondage en date, $n^{\circ} 11$ (12 août 2013). Jusqu'en 1990: RFA Ouest, depuis 1991 : ensemble de la RFA. Différence sur le total de 100\% : réponses « à la même vitesse que jusqu'ici » et nsp.

De 1982, année où la question suivante avait été posée pour la première fois, à 1988 , les Allemands de l'Ouest ont toujours affirmé à une nette majorité souhaiter des progrès plus rapides : "A quelle vitesse devrait se faire l'évolution vers une Europe unie? Plus vite, moins vite, ou à la même vitesse que jusqu'ici ? ».
La jeune génération n'assimile plus l'Europe à la paix

Les questions techniques ont pris le pas sur l'idéal

Les dernières grandes étapes ont présumé de la force des Allemands 
2010/11 : une brusque chute de l'adhésion à l'Europe

Puis, regain de confiance
Or depuis le début des années 1990 domine désormais la part de ceux qui disent qu'elle devrait plutôt se faire plus lentement. En août 2013, sur l'ensemble des Allemands, $12 \%$ optaient pour la première réponse, $43 \%$ pour la deuxième (graphique 2).

\section{L’impact de la crise de la dette souveraine}

En comparaison avec le processus rampant de distanciation vis-à-vis de l'Europe, qui a pris des années, l'impact de la crise de la dette souveraine sur la vision de l'Europe des Allemands est étonnamment faible. Pourtant, en 2011 encore, tout portait à croire que l'européanisme foncier des Allemands qui perdurait malgré tout avait été sérieusement été mis à mal sous l'effet de la multiplication des informations sur les records incessants battus par la dette en Grèce, en Irlande et d'autres Etats de la zone Euro. Après l'éclatement de la crise grecque au printemps 2010, la part de ceux qui affirmaient avoir une grande confiance dans l'UE est tombée en l'espace de quelques semaines seulement de $37 \%$ à $26 \%$. La part de ceux qui partageait l'assertion " l'Europe est notre avenir » a reculé de $53 \%$ à $41 \%$ entre avril 2010 et janvier 2011.

Il est extrêmement rare, dans la science des sondages, de constater de tels revirements brutaux de l'opinion - surtout quand, comme c'était le cas ici, il s'agit de questions somme toute générales. Les taux de réponses avaient tout d'une rupture de digue. Jusqu'ici, le malaise léger mais croissant des Allemands face à la politique européenne n'avait guère pu entamer leur adhésion foncière à l'intégration. Soudain, la patience des Allemands semblait avoir atteint ses limites, et la nervosité gagnait très nettement du terrain. Au plus fort de la crise de confiance, à l'automne 2011, $62 \%$ des Allemands disaient que la situation au sein de la zone Euro les inquiète « au plus haut point » ou « beaucoup ».

Depuis néanmoins, les esprits ont largement retrouvé leur calme. Bien que les problèmes économiques et sociaux ne soient nullement réglés dans de nombreux Etats, ce qui n'est d'ailleurs pas sans inquiéter les Allemands, la vision qu'ils ont de l'Europe tend à nouveau à se rapprocher de ce qu'elle était avant qu'éclate la crise grecque.

Graphique 3. La confiance dans l'UE croît à nouveau.

Question : "A quel point avez-vous confiance dans l'Union européenne ? Diriez-vous très grande confiance, grande confiance, pas très grande confiance, pas beaucoup, pas confiance du tout ? » (Réponses en \%)

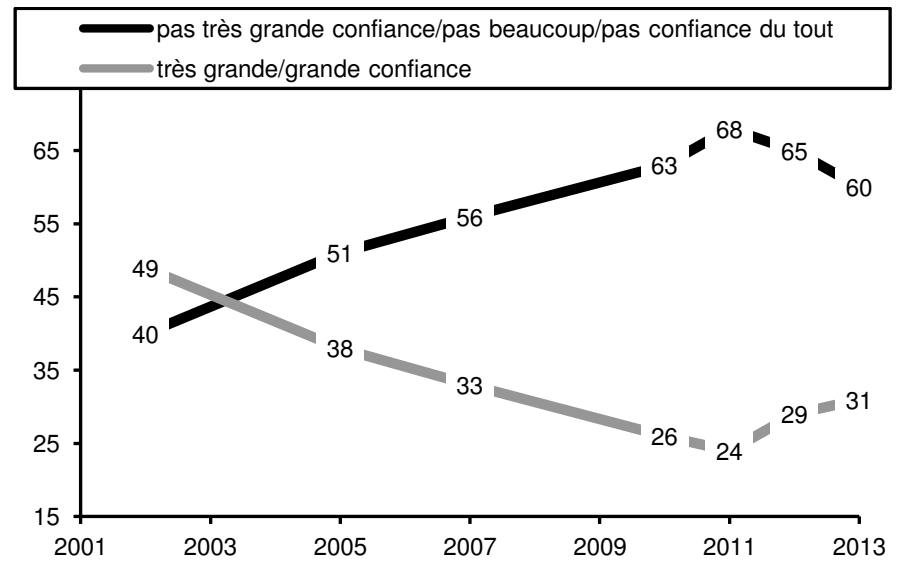

Source : Allensbacher Archiv, IfD-Umfragen, dernier sondage: $n^{\circ} 11012$ (août 2013).

On le voit par exemple aux réponses à la question : « $A$ quel point avez-vous confiance dans l'Union européenne ? ». En 2011, $68 \%$ des sondés répondaient «pas beaucoup » ou «pas du tout ». En août 2013, ils étaient $60 \%$, soit moins qu'en 2010. La part de ceux qui disaient avoir grande ou très grande confiance dans l'UE a progressé, passant de $24 \%$ à $31 \%$. Si la défiance des Allemands 
reste certes très présente, elle n'en est pas moins retombée à son niveau d'avant la crise grecque (graphique 3 ).

Ce qui, en 2010 et 2011, avait l'air d'une vague de fond se révèle a posteriori une simple et brève poussée de fièvre. C'est ce qu'illustrent par exemple les réactions à la question: "Quand vous entendez dire: 'L'Europe, c'est notre avenir', diriez-vous 'c'est vrai' ou est-ce faux à votre avis ? ” Depuis 1997, l'année où cette question fut posée pour la première fois, une nette majorité avait toujours répondu « c'est vrai ». Mais après une chute à $41 \%$ en 2011 , le taux de réponses revenait à $57 \%$ en 2012, puis restait au même niveau $(55 \%)$ en juillet 2013 (graphique 4). L'alerte de 2011 s'est révélée passagère.

\section{Graphique 4. L'avenir de l'Europe.}

Question : "Quand vous entendez dire : 'L'Europe, c'est notre avenir', diriez-vous 'c'est vrai' ou est-ce faux à votre avis ? » (Réponses en \%)

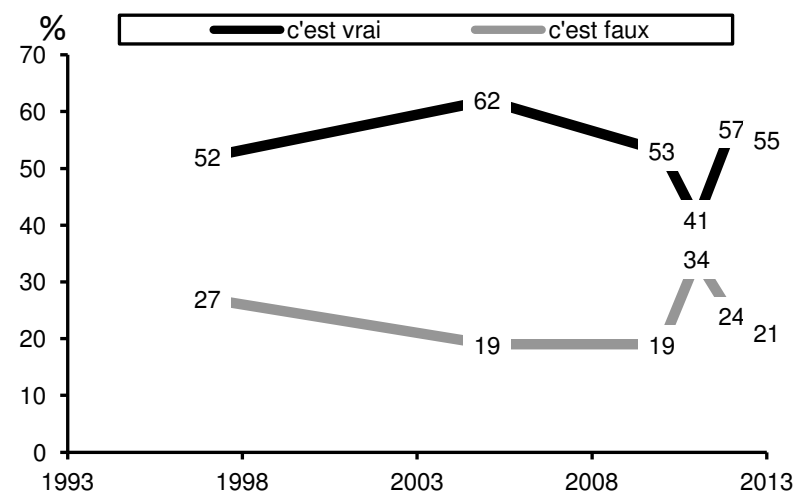

Source: Allensbacher Archiv, IfD-Umfragen, dernier sondage : n 11010 (juillet 2013).

II est frappant de constater que, dans les années où il était souvent question d'une „crise de l'Euro“, la réputation de l'Euro non seulement n'a pas souffert durablement, mais que, au contraire, elle s'est nettement consolidée. Certes, de 2009 à 2011, la part des Allemands disant avoir grande confiance en l'Euro est tombé d'un niveau exceptionnellement élevé de $44 \%$ à seulement $17 \%$; mais en 2013, elle était remontée à $28 \%$, c'est-à-dire à son niveau habituel. Plus remarquables encore sont les réponses à la question : "Préféreriez-vous repasser au deutsche mark, ou ne diriez-vous pas cela ?».

Graphique 5 : La nostalgie du deutsche mark recule.

Question : "Préféreriez-vous repasser au deutsche mark, ou ne diriez-vous pas cela ? » (Réponses en \%)

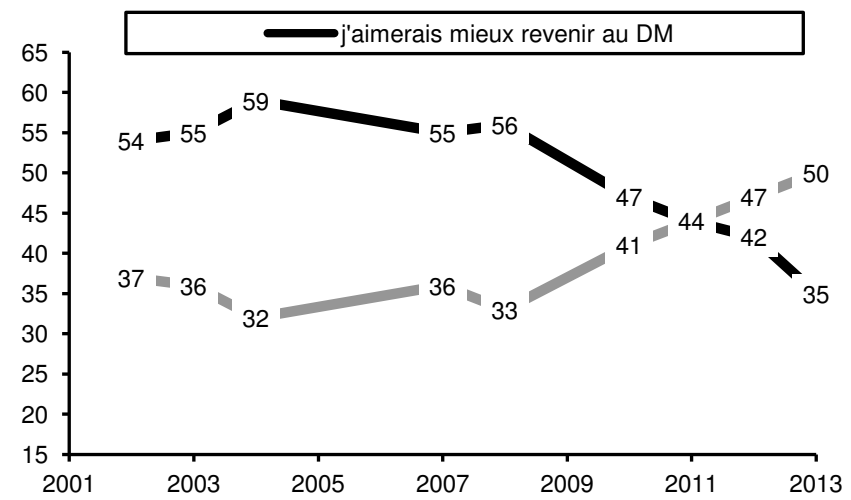

Source : Allensbacher Archiv, IfD-Umfragen, dernier sondage : n 11010 (juillet 2013).

Depuis 2002, où la question avait été posée pour la première fois, une nette majorité des sondés avait toujours répondu qu'ils préféreraient le deutsche mark à l'Euro. Or il n'en fut pas de même en 2011, année particulièrement critique, où la part de ceux qui ne rêvaient pas de la réintroduction de l'ancienne
La perte de confiance dans l'Europe était passagère

Les Allemands ont confiance dans l'Euro 
L’intégration européenne tient à cœur aux Allemands

L'image de l'Europe a pris des contours plus précis... monnaie nationale se révéla identique à celle des adeptes du deutsche mark : $44 \%$. En juillet $2013,50 \%$ des sondés disaient ne pas rêver du retour du deutsche mark. Un peu plus d'un tiers seulement (35\%) affirmait le contraire (graphique 5). Ces réponses concordent avec l'optimisme des Allemands, lorsqu'ils disent que l'avenir de l'Euro est assuré. A la question : "Quelle est votre appréciation ? L'Euro existera-t-il toujours dans dix ans ou en doutez-vous ? ", $51 \%$ d'entre eux répondaient, toujours en juillet 2013, qu'à leur avis, la monnaie unique existerait toujours dans dix ans ; $39 \%$ en doutaient.

II s'en dégage l'impression que, malgré le scepticisme des Allemands au vu des risques budgétaires et financiers inhérents aux mécanismes de sauvetage des Etats en crise dans la zone Euro, l'intégration européenne leur tient à nouveau plus à cœur qu'avant. Comment s'explique cette évolution ? Les réponses à une question où le sondeur demande aux personnes interrogées de désigner sur la liste présentée les points qu'ils associent avec l'UE sont révélatrices à cet égard. Cette question avait été posée d'abord en 2006, puis en 2013. La comparaison des réponses montre que, en 2013, les sondés désignaient la plupart des points plus souvent que sept ans auparavant, aussi bien les points négatifs que positifs.

Ainsi, en 2006, $40 \%$ des Allemands affirmaient que, pour eux, l'UE s'assimile à une bureaucratie débordante ; à l'été 2013, ils étaient $60 \%$. En 2006, $32 \%$ y associaient la volonté de tout réglementer, $60 \%$ sept ans plus tard. Or dans le même temps, on observe aussi une hausse de la part de ceux pour qui l'UE évoque la sécurité de ne plus voir éclater de guerre en Europe (55\%, puis $62 \%$ ) ou qui partagent l'assertion "une Europe sans frontières, où on peut voyager et exercer son métier sans entraves " (62\%, puis $72 \%)$. Quand on additionne toutes les assertions négatives, toutes les positives, et qu'on calcule la moyenne, on s'aperçoit d'un net changement : entre 2006 et 2013, le taux moyen de toutes les associations positives est passé de $49 \%$ à $53 \%$, celui des associations négatives, de $40 \%$ à $59 \%$ (graphique 6 ). On voit là d'une part que l'image de l'Europe a certes pris globalement des teintes négatives, la hausse étant plus nette dans le choix des assertions négatives, mais on s'aperçoit aussi - et ce constat est plus significatif encore - que les contours de l'image de l'Europe se sont fait plus précis.

\section{Graphique 6. La représentation de l'Europe gagne en précision.}

Question: “Nous avons inscrit sur cette liste ce que d'autres nous ont dit que l'Union européenne évoque pour eux. Parmi ces points, desquels diriez-vous que vous aussi, vous les associez à l'UE ? " (présentation de la liste). (Réponses en \%)

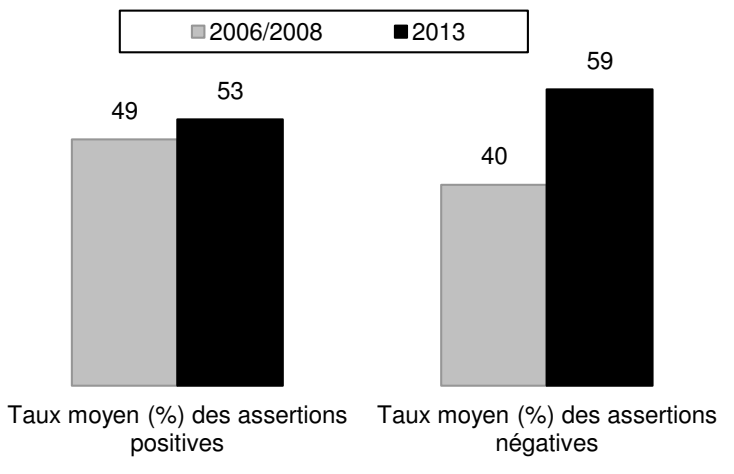

Source : Allensbacher Archiv, IfD-Umfragen n 7069, 10020, 11010.

.. grâce à la crise de la dette souveraine

Le sens à accorder à un tel profil de réponses est bien connu dans le domaine des sondages sur les biens de consommation. La différence fondamentale entre les marques à forte et faible identité réside dans le fait que les premières ne suscitent souvent chez les consommateurs pas d'associations foncièrement différentes que les secondes, mais ces associations sont alors plus vivantes, plus colorées. C'est à la lumière de ces enseignements qu'il faut interpréter les réponses sur l'Union européenne. Elles montrent dès lors que la crise de la 
dette souveraine a hissé plus qu'avant le thème de l'Europe dans la conscience des Allemands. Et comme dans le cas des marques à forte identité, les représentations liées à l'Europe, qui étaient longtemps restées floues, ont gagné en clarté, prenant une plasticité vivante - aussi bien sur leurs aspects négatifs que positifs. Ainsi, paradoxalement, la crise de la dette souveraine a en un certain sens renforcé l'image de l'Europe qu'ont les Allemands.

De même, l'hypothèse que nous formulions au début - à savoir que la crise au sein de l'UE aurait eu pour effet que l'intégration européenne soit perçue comme une menace pour la liberté individuelle - se révèle sans fondement. Quand on demande directement aux Allemands si le processus d'intégration leur apporte plus ou moins de liberté, plus ou moins de prospérité, de justice sociale ou de sécurité, les réponses ne sont pas univoques. En août 2013, une nette majorité relative des sondés affirmait que ce processus se soldait par moins de prospérité et moins de justice sociale pour les Allemands. Mais dans le même temps, une nette majorité relative d'entre eux affirmait qu'il leur apportait plus de sécurité et plus de liberté (graphique 7).

\section{Graphique 7. Effets supposés de l'intégration européenne.}

Question: "Diriez-vous que l'intégration européenne apporte aux Allemands globalement plus de liberté/prospérité/justice sociale/sécurité ou au contraire moins de liberté/prospérité/justice sociale / sécurité, ou alors ne change-t-elle pas grand-chose ? " (formulation des questions déclinée en quatre combinaisons). (Réponses en \%)

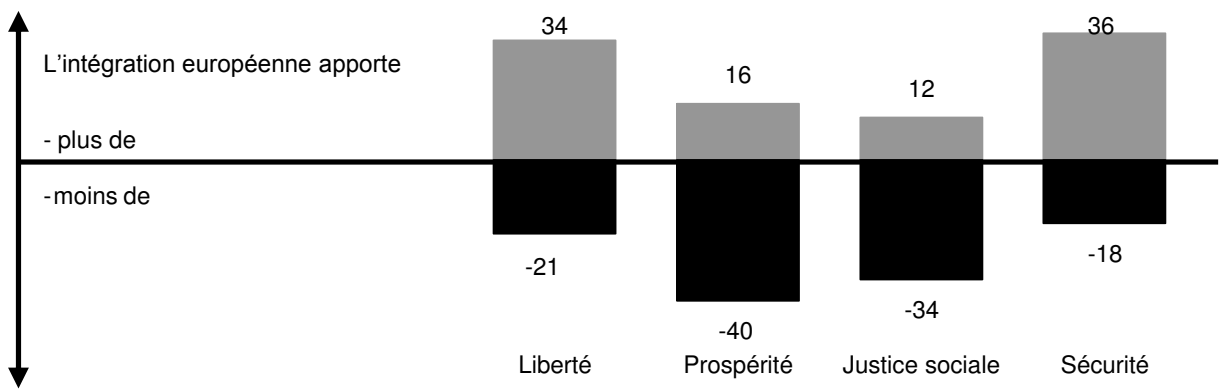

Source : Allensbacher Archiv, IfD-Umfrage n¹1012, août 2013.

Il est particulièrement frappant de constater que ceux qui voient l'intégration européenne comme un facteur renforçant la liberté se recrutent très largement parmi ceux qui accordent généralement le plus de prix à la liberté. Ceux qui, à d'autres moments de l'entretien, affirmaient que la liberté leur importait plus que l'équité des chances ou la justice sociale étaient nettement plus nombreux à dire que l'intégration européenne apportait plus de liberté aux Allemands que ceux qui préféraient l'équité des chances à la liberté (graphique 8).

\section{Graphique 8. L'intégration européenne apporte-t-elle plus de liberté ?}

Question: "Diriez-vous que l'intégration européenne apporte aux Allemands globalement plus de liberté ou moins de liberté ou que cela reste du pareil au même, qu'elle ne change pas grand-chose ? " (Réponses en \%)

plus de liberté moins de liberté

41

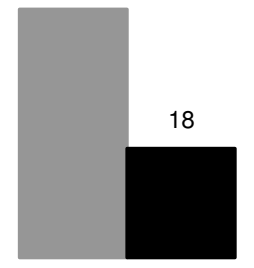

Sondés préférant la liberté à l'équité des chances

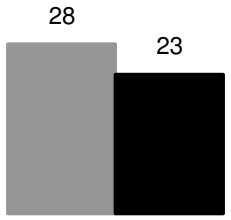

Sondés préférant l'équité des chances à la liberté

Source : Allensbacher Archiv, IfD-Umfrage n¹1012, août 2013. 
Ces réponses réfutent l'idée selon laquelle la praxis du processus d'intégration européenne nourrirait l'inquiétude de ceux qui accordent un grand prix à la liberté individuelle. C'est bien le contraire qu'on peut constater : ceux qui partagent cette inquiétude ne représentent qu'une petite minorité parmi ceux qui accordent une grande importance à la liberté.

A PREMIÈre VUE, L'ATTITUDE deS ALLEMANDS VIS-À-VIS DE L'UNION EUROPÉENNE peut sembler contradictoire : d'un côté ils se plaignent de la soi-disant volonté des instances communautaires de tout réglementer, de l'autre, ils considèrent I'UE plus comme alimentant la liberté que comme une instance la réduisant. Rien dans ces attitudes ne laisse entrevoir que des mouvements protestataires comme le parti AfD en Allemagne puissent séduire les masses. Comment s'explique cette contradiction?

On comprend mieux le pourquoi des réponses des sondés quand on a conscience du fait que le reproche fait à l'UE d'être trop bureaucratique n'est pas en réalité ce qu'il semble être à première vue. Dans les débats publics, les lamentations sur l'ingérence de l'Europe dans la législation nationale s'accumulent, les références à l'interdiction des ampoules à incandescence et la réglementation sur la courbure des concombres sont entrés dans l'imagerie populaire au point qu'on imagine qu'il s'agirait là des symptômes d'une profonde et authentique indignation. Or les sondages sont loin de confirmer cette impression.

Depuis au moins deux décennies, la formation de l'opinion publique sur l'intégration européenne s'effectue au sein de deux sphères qu'il convient de bien séparer si on veut comprendre l'attitude des Allemands vis-à-vis de l'Europe. On pourrait également parler dans ce contexte de la double image de l'Europe qu'ont les Allemands.

Ces deux sphères sont d'une part celle du quotidien de l'actualité politique et, indépendamment de la première, d'autre part celle de l'approche fondamentale de l'Europe et de l'UE. La première sphère est celle qui prévaut en matière d'information des médias et dans les conversations de la vie quotidienne. Elle est empreinte d'aspects négatifs. Mais en dessous de cette surface se décèle la deuxième sphère, se caractérisant, elle, par une attitude positive d'une remarquable stabilité. Cette position fondamentale, sans lien avec la première sphère, ne se nourrit donc pas essentiellement de considérations budgétaires, financières ou géopolitiques, mais du vague sentiment d'une identité européenne que la crise de la finance mondiale semble non seulement avoir laissé indemne, mais aussi et bien au contraire renforcé.

Ainsi, dans les années précédant la crise de la finance mondiale, les Allemands s'intéressaient nettement moins en tendance à l'intégration européenne. Aujourd'hui, le changement est flagrant. Tout se passe comme si la crise avait brutalement réveillé les Allemands et, malgré tous les soucis qu'elle a apportés, avait également aiguisé leur prise de conscience des risques encourus si l'intégration européenne échouait. C'est ainsi que, en août 2013, 60 \% des Allemands approuvaient l'assertion: "Malgré toutes les difficultés que nous rencontrons actuellement en Europe, nous autres Européens faisons, au fond, partie d'une même famille ". Inutile de nier l'évidence : dans la hiérarchie des positions face à l'Europe, cette vision-là prévaut de loin sur la grogne épidermique que peuvent susciter au jour le jour les mécanismes de sauvetage de la zone Euro ou autres courbures réglementaires des concombres.

(Traduction : I. Bourgeois)

\section{Indications bibliographiques}

- AdenAUER K., Erinnerungen 1945-1953, Stuttgart, 1965

- Bernhard H., Goetz W., Wiegler P. (eds), Gustav Stresemann. Vermächtnis. Der Nachlass in drei Bänden, vol. 3, Berlin, 1932

- MitterRand F., De l'Allemagne, de la France, Paris, 1996

- Petersen T., "Le difficile rapport des Allemands à la liberté », et "Une nouvelle identité allemande? ", Regards sur l'économie allemande, n¹07, hiver 2012 et $n^{\circ} 91$, mai 2009. 